\title{
Stepwise In-straw Dilution and Direct Transfer Using Open Pulled Straws (OPS) in the Mouse: A Potential Model for Field Manipulation of Vitrified Embryos
}

\author{
Qi-En YANG ${ }^{1)}$, Yun-Peng HOU ${ }^{1)}$, Guang-Bin ZHOU ${ }^{1,3)}$, Zhong-Qiang YANG ${ }^{1)}$ \\ and Shi-En $\mathrm{ZHU}^{1,2)}$ \\ ${ }^{1)}$ Laboratory of Animal Embryonic Biotechnology, ${ }^{2)}$ State Key Laboratory of \\ Agrobiotechnology, College of Animal Science and Technology, China Agricultural University, \\ Beijing 100094 and ${ }^{3)}$ Dujiangyan campus of Sichuan Agricultural University, Dujiangyan
} 611830, P.R. China

\begin{abstract}
In the present study, mouse blastocysts were employed to investigate the feasibility and efficiency of stepwise in-straw dilution and direct transfer using the open pulled straw (OPS) method. In experiment I, the effects of various vitrification solutions (VS) on embryo survival were examined. After thawing, the expanded blastocyst rates $(97.59$ and $95.05 \%)$ and hatching rates $(80.48$ and $78.95 \%)$ achieved in the EDFS30 [15\% ethylene glycol (EG), 15\% dimethyl sulfoxide (DMSO), Ficoll, and sucrose] and EFS40 [40\% EG, Ficoll, and sucrose] groups were no different from those $(96.15 \%$ and $83.33 \%$ ) of the control group. However, the rates in the EFS30 [30\% EG, Ficoll, and sucrose] (87.80 and $55.43 \%$ ) and EDFS40 [20\% EG, 20\% DMSO, Ficoll, and sucrose] (95.69 and 70.97\%) groups were significantly lower than those $(96.15$ and $83.33 \%)$ of the control group $(\mathrm{P}<0.05)$. In the experiment II, the effects of the volume of VS in the OPS on the survival of embryos after in-straw thawing were investigated. When the length of the VS in the column was less than $1 \mathrm{~cm}$, the in vitro viability of embryos thawed by stepwise in-straw dilution was no different among the experimental and control groups. The embryos could be successfully thawed by immersing the OPS in $0.5 \mathrm{M}$ sucrose for $3 \mathrm{~min}$ and then $0.25 \mathrm{M}$ sucrose for $2 \mathrm{~min}$. In experiment III, the effect of immersion time of the OPS in diluent (PBS) on the viability of vitrified embryos was investigated. After in-straw thawing, OPSs were immersed immediately in $1 \mathrm{ml}$ PBS for 0 to $30 \mathrm{~min}$. When the immersion time of the OPSs in PBS was less than $12 \mathrm{~min}$, in vitro development of the in-straw thawed embryos was no different from that of the controls. In experiment IV, in-straw thawed blastocysts were directly transferred to pseudopregnant mice to examine their in vivo developmental viability. The pregnancy $(91.67 \%)$ and birth rates $(42.42 \%)$ of embryos in-straw thawed and directly transferred were no different from those of the unvitrified controls ( 90.90 and $40 \%$ ) and embryos thawed by the conventional method (84.61 and $46.94 \%$ ). These results demonstrate that mouse embryos vitrified with OPS could be successfully thawed by stepwise in-straw dilution and transferred directly to a recipient and that this method might be a model for field manipulation of vitrified embryos in farm animals.
\end{abstract}

Key words: Blastocyst, In-straw dilution, Mouse, Open pulled straw (OPS), Vitrification

(J. Reprod. Dev. 53: 211-218, 2007)

Accepted for publication: October 14, 2006

Published online: November 29, 2006

Correspondence: S. Zhu (e-mail: zhushien@cau.edu.cn) itrification, an efficient approach to cryopreservation, has been applied in laboratories to mammalian embryos [1], the oocytes and embryos of domestic animals [2], and the oocytes and 
embryos of humans [3]. Compared with the conventional freezing method, vitrification is relatively simple, rapid, and inexpensive. During freezing, high cooling rates are achieved by plunging containers into liquid nitrogen, and ice crystal formation is avoided by supplementation with highly concentrated cryoprotectants. Therefore, the vitrification method can be applied successfully to cryopreserve goat oocytes at the germinal vesicle stage [4] and porcine embryos [5] that have proven to be very sensitive to cryoinjury caused by the conventional protocol.

To improve the efficiency of the vitrification method, different cryoprotectants and vitrification tools have been studied. The most commonly used cryoprotectants for mammalian embryo vitrification are limited to five reagents, including DMSO, glycerol, ethylene glycol, propylene glycol and acetamide [1]. One of the most effective containers for vitrification is the OPS [6], which has been used successfully for vitrification of embryos and oocytes [2].

Meanwhile, to simplify the thawing and transferring procedure and make it more suitable for field use, some researchers have examined the feasibility of in-straw rehydration and direct transfer in the bovine [7], rabbit [8], ovine [9, 10] and equine [11]. One feature of this thawing procedure is in-straw dilution, embryo thawing, and direct transfer of the thawed embryos. This instraw dilution and direct transfer method reduces the requirements for equipment and technical skill, which are obstacles to application of embryo transfer technology at many farms where the appropriate conditions are not available. Therefore, this modified method could be more suitable for wide application under field conditions.

Recently, some studies concerning direct thawing and transfer of embryos vitrified in OPSs have been reported $[12,13]$. However, details essential for successfully thawing and transferring vitrified embryos were not provided, for instance, the volume of VS in the OPS and the optimal time for successful thawing. Moreover, these previous reports were limited to a one-step dilution, which is less effective in reducing osmotic injury than stepwise dilution [14, 15].

Therefore, the present research was designed to investigate different cryoprotectants, different volumes of vitrification solution (VS) in the OPS, and different immersion times in the dilution solution on the viability of vitrified mouse blastocysts. This study will provide the basic technical parameters for in-straw manipulation of vitrified embryos for other laboratories and for farm animals under field conditions.

\section{Materials and Methods}

The hormones (eCG and hCG) used for superovulation were purchased from Huafu Advanced Biotechnology Company (Tianjin, P. R. China). Unless otherwise stated, all other chemicals for the present study were purchased from Sigma Company (Sigma Chemical, St. Louis, MO, USA). Embryo handling was performed in the laboratory at ambient temperature $(25 \pm 0.5 \mathrm{C})$; all vitrification media, diluents, and embryos were maintained at $37 \mathrm{C}$ on a hot plate.

\section{Preparation of solutions}

Pretreatment media: PBS was supplemented with $10 \%(\mathrm{v} / \mathrm{v})$ EG or $10 \%$ EG and $10 \%$ DMSO to be used as pretreatment media.

Vitrification solutions: The vitrification solutions used in this study included EFS30 containing 30\% (v/v) EG in FS solution and EFS40 containing 40\% (v/v) EG in FS solution, as described by Zhu et al. [15] and Kasai et al. [16]. EDFS30 [17] containing $15 \%(\mathrm{v} / \mathrm{v})$ EG and 15\% (v/v) DMSO in FS solution and EDFS40 containing 20\% (v/v) EG and 20\% (v/ v) DMSO in FS solution were also used. The FS solution consisted of PBS medium containing 300 $\mathrm{g} / 1$ Ficoll and $171.2 \mathrm{~g} / 1$ sucrose.

Diluents: PBS was supplemented with $0.5 \mathrm{M}$ or $0.25 \mathrm{M}$ sucrose (0.5 MS or 0.25 MS) to be used as diluent.

\section{Manufacture of OPSs}

Pulled straws were manufactured as described by Vajta et al. (1997) with the following modifications. The plugs of $0.25-\mathrm{ml}$ plastic straws (IMV, L'Aigle, France) were removed, and the straws were heat-softened at the midpoint over a hot plate and pulled manually. The pulled straws were cut at the tapered end with a surgical blade. The inner diameter of the tip was $0.15-0.19 \mathrm{~mm}$, and the wall thickness was no greater than $0.05 \mathrm{~mm}$ (measured with a microforge; MF900; Narishige, Tokyo, Japan). The thin part of each OPS was 
approximately $2.5 \mathrm{~cm}$ long.

\section{Embryo collection}

Kunming (KM) mice (Academy of Military Medical Sciences, Beijing, P. R. China) were kept in a room at $20 \pm 2 \mathrm{C}$ under a $14 \mathrm{~h}$ (1)0600-2000 h) light and $10 \mathrm{~h}$ (2000-(1)0600 h) dark schedule. Female mice 7 to 11 weeks of age were induced to superovulate by i.p. injection of $10 \mathrm{IU}$ of eCG followed by $10 \mathrm{IU}$ of hCG $48 \mathrm{~h}$ later. The following morning (designated day 1 of pregnancy), the females were examined for mating as determined by the presence of a vaginal plug. Mated females were euthanized by cervical dislocation at 88 to 90 $\mathrm{h}$ after hCG injection to collect blastocysts. The embryos were recovered by flushing the excised uteri with PBS medium. Some of the blastocysts were cultured at $37 \mathrm{C}$ with $5 \% \mathrm{CO}_{2}$ in humidified air in an incubator for $40 \mathrm{~h}$ to collect hatching blastocysts in mCZB medium [18]. Embryos with normal morphology were used for the cryopreservation experiments. The blastocysts were defined as having morphologically survived if the embryos possessed an intact zona pellucida and refractive blastocoel.

\section{Experimental design}

Experiment I: Effects of different vitrification solutions on the in vitro development of OPSvitrified mouse blastocysts

Vitrification of embryos: In vivo derived blastocysts were vitrified with various types of VS in open pulled straws. The embryos were pretreated in $10 \% \mathrm{EG}+10 \% \mathrm{DMSO}$, or $10 \% \mathrm{EG}$ for $30 \mathrm{sec}$, loaded into EDFS30, EDFS40, EFS30, or EFS40 in the narrow end of an OPS, and left for 25 sec. Then, the straws were immediately plunged into liquid nitrogen $\left(\mathrm{LN}_{2}\right)$. Five to six embryos were loaded into each OPS.

Thawing by conventional method: After storage for at least 7 days in liquid nitrogen, the embryos were thawed for in vitro culture. During thawing, OPSs containing embryos were kept at room temperature for $1 \mathrm{sec}$ to remove the liquid nitrogen, and then the narrow tip was immersed in $500 \mu \mathrm{l}$ of 0.5 MS. The embryos were expelled from the straws into medium. The embryos were transferred into another $100 \mu$ l of 0.5 MS under oil after $0.5 \mathrm{~min}$ and were kept there for $2.5 \mathrm{~min}$. Subsequently, there were transferred into $0.25 \mathrm{MS}$ and kept there for $2 \mathrm{~min}$. Finally, the embryos were washed three times with PBS. The recovered embryos were cultured in modified CZB [18] (mCZB) at $37 \mathrm{C}$ in $5 \% \mathrm{CO}_{2}$ in humidified air. After 24 or $48 \mathrm{~h}$ of culture, the number of embryos that developed to expanded or hatching blastocysts was recorded.

Experiment II: Effects of different volumes of vitrification solution in OPSs on the in vitro development of vitrified/stepwise in-straw thawed embryos

Embryo vitrification: The narrowed section of each OPS was labeled with lengths of $0.3,0.5,0.7$, $1.0,1.2,1.5,1.7$ and $2.0 \mathrm{~cm}$ starting from the narrow tip using different marker colors to designate the volume of VS loaded into the OPS. The VS (EDFS30) with the highest in vitro survival of treated embryos in experiment I was used for embryo vitrification in experiment II. Embryos were loaded with various volumes of vitrification solution into the narrow section of a pulled straw and vitrified as described above.

Thawing using a new stepwise in-straw dilution method: After storage, the cryoprotectants were diluted by a new method in which an OPS was used as a container for dilution (Fig. 1, left).

Two modified tubes (the top half of $15 \mathrm{ml}$ tubes was cut off) containing 0.5 MS and 0.25 MS were placed upright in $37 \mathrm{C}$ water bath (Fig.1, right). The modified tubes will hereafter be referred to solely as "tubes". The OPS was immersed and left to stand in a tube containing $0.5 \mathrm{MS}$ for $3 \mathrm{~min}$ and then $0.25 \mathrm{MS}$ for $2 \mathrm{~min}$. The embryos were expelled into PBS and washed twice for in vitro culture. Embryos thawed by the conventional method (as described in the Materials and methods section of this paper) were used as a control.

Experiment III: Effect of immersion time in PBS on the in vitro development of in-straw thawed blastocysts

Based on the data obtained from experiment II, embryos were vitrified with the optimal volume of VS and thawed as described above. After dilution, OPSs containing thawed blastocysts were transferred and left to stand in tubes containing PBS for $0,2,3,4,6,8,10,12,15,20$ or $30 \mathrm{~min}$, respectively. Then, the embryos were recovered and cultured for examination of their viability. Embryos thawed by the conventional method (as described in the Materials and methods section of this paper) were used as a control.

Experiment IV: In vivo development of in-straw 

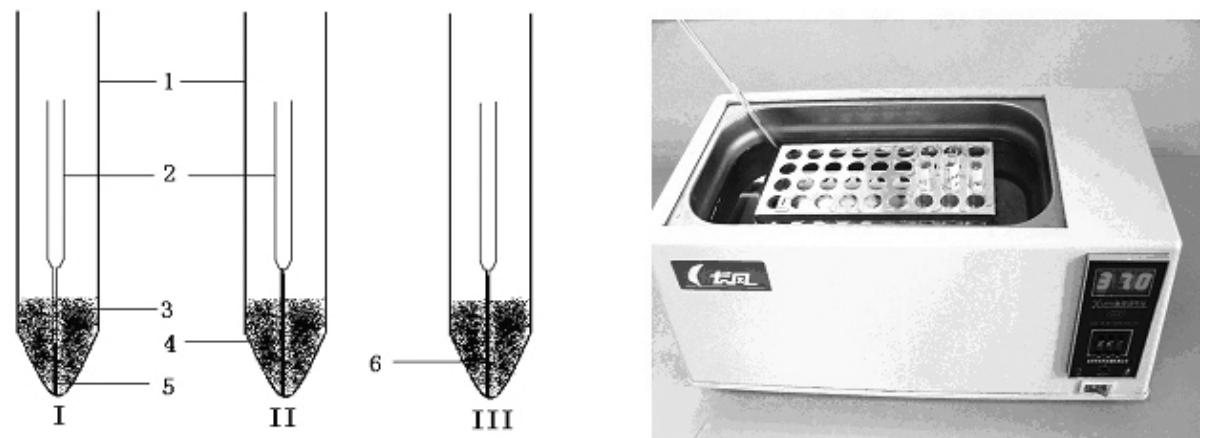

Fig. 1. The simple equipment required for in-straw dilution of embryos vitrified by the OPS method. Left side: (1) Tube containing sucrose solutions or PBS, (2) OPS, (3) 0.5 MS, (4) 0.25 MS, (5) Vitrification medium containing embryos, and (6) PBS. Right side: a 37 C bath chamber.

Table 1. In vitro development of mouse blastocysts vitrified with different solutions and thawed by the conventional method

\begin{tabular}{|c|c|c|c|c|c|c|c|}
\hline \multicolumn{2}{|c|}{ Pretreatment } & \multicolumn{2}{|c|}{ Vitrification } & \multirow[t]{2}{*}{ Vitrified } & \multirow{2}{*}{$\begin{array}{c}\text { Recovered } \\
(\%)\end{array}$} & \multicolumn{2}{|c|}{ Developed to } \\
\hline Solution & Time & Solution & Time & & & $\mathrm{EB}^{*}(\%)$ & $\mathrm{HB}^{*}(\%)$ \\
\hline $10 \% \mathrm{E}+10 \% \mathrm{D}$ & $30 \mathrm{sec}$ & EDFS30 & $25 \mathrm{sec}$ & 82 & $82(100)$ & 81 (97.59) & $66(80.48)^{\mathrm{AB}}$ \\
\hline $10 \% \mathrm{E}+10 \% \mathrm{D}$ & $30 \mathrm{sec}$ & EDFS40 & $25 \mathrm{sec}$ & 93 & $93(100)$ & 89 (95.69) & $66(70.97)^{\mathrm{B}}$ \\
\hline $10 \% \mathrm{EG}$ & $30 \mathrm{sec}$ & EFS30 & $25 \mathrm{sec}$ & 82 & $82(100)$ & $72(87.80)$ & $45(55.43)^{\mathrm{C}}$ \\
\hline $10 \% \mathrm{EG}$ & $30 \mathrm{sec}$ & EFS40 & $25 \mathrm{sec}$ & 76 & $76(100)$ & $73(95.05)$ & $60(78.95)^{\mathrm{AB}}$ \\
\hline Control & - & - & - & 78 & 78 (100) & $75(96.15)$ & $65(83.33)^{\mathrm{A}}$ \\
\hline
\end{tabular}

This experiment was repeated 6 times. Values with different superscripts within the same column are significantly different $(\mathrm{P}<0.05)$.

*EB=expanded blastocyst; $\mathrm{HB}=$ hatched blastocyst.

thawed blastocysts after direct transfer

This experiment examined the in vivo development of blastocysts vitrified and thawed by this in-straw dilution method. After in-straw dilution in tubes, OPSs were left to stand in PBS for $6 \mathrm{~min}$. Then, the OPSs containing embryos were used as catheters for transferring embryos into each uterine horn of recipients (12 embryos per animal) that had mated with a vasectomized male 4 days previously. Fresh embryos and vitrified embryos that were thawed by the conventional method (as described in the Materials and methods section of this paper) were transferred to recipients as controls.

\section{Statistical analysis}

All experiments were repeated at least three times. Data were subjected to arcsine transformation prior to ANOVA (PROC GLM; SAS Institute, Cary, NC, USA). Statistically significant differences between treatment groups $(\mathrm{P}<0.05)$ were determined using the Tukey Test. A P value of less than 0.05 was considered to be statistically significant.

\section{Results}

\section{Experiment I}

As shown in Table 1, no significant differences $(\mathrm{P}>0.05)$ were detected for the expanded blastocyst rates between the treated $(87.80-97.59 \%)$ and control (95.16\%) groups. There were no significant differences in hatching blastocyst rates between the control $(83.3 \%)$ and EDFS30 $(80.48 \%)$ or EFS40 $(78.95 \%)$ groups; however, the hatching blastocyst rates of the EDFS40 and EFS30 groups were significantly lower than that of the control group $(\mathrm{P}<0.05)$.

\section{Experiment II}

There was no difference in the in vitro viability of 
Table 2. Effect of length of vitrification solution in the OPS on in vitro development of vitrified mouse blastocysts after the new stepwise in-straw dilution method

\begin{tabular}{ccccc}
\hline Length $(\mathrm{cm})$ & Vitrified & Recovered $(\%)$ & \multicolumn{2}{c}{ Developed to } \\
\cline { 3 - 5 } & & & $\mathrm{EB}^{*}(\%)$ & $\mathrm{HB}^{*}(\%)$ \\
\hline 0.3 & 66 & $66(100)$ & $66(100)^{\mathrm{A}}$ & $52(78.78)^{\mathrm{A}}$ \\
0.5 & 73 & $73(100)$ & $73(100)^{\mathrm{A}}$ & $55(75.34)^{\mathrm{A}}$ \\
0.7 & 75 & $75(100)$ & $75(100)^{\mathrm{A}}$ & $57(76.00)^{\mathrm{A}}$ \\
1.0 & 79 & $79(100)$ & $79(100)^{\mathrm{A}}$ & $61(77.21)^{\mathrm{A}}$ \\
1.2 & 74 & $74(100)$ & $59(79.72)^{\mathrm{AB}}$ & $43(58.11)^{\mathrm{B}}$ \\
1.5 & 79 & $79(100)$ & $42(53.16)^{\mathrm{BC}}$ & $18(22.74)^{\mathrm{C}}$ \\
1.7 & 72 & $72(100)$ & $50(69.44)^{\mathrm{C}}$ & $20(27.77)^{\mathrm{C}}$ \\
2.0 & 65 & $65(100)$ & $26(40.00)^{\mathrm{C}}$ & $11(16.92)^{\mathrm{C}}$ \\
Control & 67 & $67(100)$ & $67(100)^{\mathrm{A}}$ & $53(79.10)^{\mathrm{A}}$ \\
\hline
\end{tabular}

This experiment was repeated 6 times. Values with different superscripts within the same column are significantly different $(\mathrm{P}<0.05)$.

${ }^{*} \mathrm{~EB}=$ expanded blastocyst; $\mathrm{HB}=$ hatched blastocyst.

Table 3. In vitro development of in-straw thawed blastocysts after storage in PBS for different amounts of time

\begin{tabular}{ccccc}
\hline \multirow{2}{*}{ Time (min) } & Vitrified & Recovered (\%) & \multicolumn{2}{c}{ Developed to } \\
\cline { 4 - 5 } & & & EB $^{*}(\%)$ & HB $^{*}(\%)$ \\
\hline 0 & 60 & $60(100)$ & $60(100)^{\mathrm{A}}$ & $47(78.33)^{\mathrm{A}}$ \\
2 & 60 & $60(100)$ & $60(100)^{\mathrm{A}}$ & $45(75.00)^{\mathrm{A}}$ \\
3 & 70 & $70(100)$ & $70(100)^{\mathrm{A}}$ & $50(71.42)^{\mathrm{A}}$ \\
4 & 65 & $65(100)$ & $65(100)^{\mathrm{A}}$ & $47(72.31)^{\mathrm{A}}$ \\
6 & 71 & $71(100)$ & $71(100)^{\mathrm{A}}$ & $52(73.24)^{\mathrm{A}}$ \\
8 & 72 & $72(100)$ & $71(98.61)^{\mathrm{A}}$ & $51(70.83)^{\mathrm{A}}$ \\
10 & 63 & $63(100)$ & $63(100)^{\mathrm{A}}$ & $44(69.84)^{\mathrm{A}}$ \\
12 & 62 & $62(100)$ & $62(100)^{\mathrm{A}}$ & $46(74.19)^{\mathrm{A}}$ \\
15 & 64 & $64(100)$ & $64(100)^{\mathrm{A}}$ & $33(51.56)^{\mathrm{B}}$ \\
20 & 63 & $63(100)$ & $61(98.82)^{\mathrm{A}}$ & $28(45.90)^{\mathrm{BC}}$ \\
30 & 62 & $62(100)$ & $48(77.42)^{\mathrm{B}}$ & $19(30.64)^{\mathrm{C}}$ \\
Control & 67 & $67(100)$ & $67(100)$ & $53(79.10)^{\mathrm{A}}$ \\
\hline
\end{tabular}

This experiment was repeated 6 times. Values with different superscripts within the same column are significantly different $(\mathrm{P}<0.05)$.

* $\mathrm{EB}=$ expanded blastocyst; $\mathrm{HB}=$ hatched blastocyst.

embryos thawed by stepwise in-straw dilution between the experimental and control groups when the length designating the volume of VS was less than $1 \mathrm{~cm}$. When the length designating the volume of VS was more than $1.2 \mathrm{~cm}$, there was no difference in the survival of embryos thawed by stepwise in-straw dilution among the experimental and control groups; however, the expanded $(<69.44 \%)$ and hatching blastocyst rates $(27.77 \%)$ of these embryos were significantly lower than those of the controls (100 and $79.10 \% ; \mathrm{P}<0.05)$ (Table 2).

\section{Experiment III}

There were no significant differences among the groups in terms of blastocyst rates or hatching blastocyst rates when the immersion time in PBS was shorter than $12 \mathrm{~min}$. When the time exceeded $15 \mathrm{~min}$, the expanded blastocyst rate was similar to that of the controls, but the hatching blastocyst rate was significantly reduced $(51.56 \%)$ compared with that of the controls $(79.10 \% ; \mathrm{P}>0.05)$ (Table 3).

\section{Experiment IV}

The pregnancy (91.67\%) and birth rates (42.42\%) of embryos in-straw thawed and directly transferred were similar to those of fresh embryos (90.90 and $40 \%$ ) and were not significantly different from the ones thawed by the conventional method 
Table 4. In vivo development of in-straw treated mouse blasocysts after direct transfer

\begin{tabular}{lccc}
\hline Treatment & \multicolumn{2}{c}{$\begin{array}{c}\text { Recipients } \\
\text { pregnant/transferred (\%) }\end{array}$} & \multicolumn{2}{c}{ Birth (\%) } \\
\cline { 3 - 4 } & $11 / 12(91.67)$ & $56 / 132(42.42)^{\mathrm{A}}$ & $56 / 144(38.89)^{\mathrm{B}}$ \\
Direct & $11 / 13(84.61)$ & $62 / 132(46.94)^{\mathrm{A}}$ & $62 / 156(39.79)^{\mathrm{B}}$ \\
Conventional & $10 / 11(90.90)$ & $48 / 120(40.00)^{\mathrm{A}}$ & $48 / 132(36.36)^{\mathrm{B}}$ \\
Nonfreezing & &
\end{tabular}

Values with different superscripts within the same column are significantly different $(\mathrm{P}<0.05)$.

(84.61 and 46.94\%) (Table 4).

\section{Discussion}

Blastocysts have widely been adopted for embryo production, cryopreservation and transfer in farm animals, especially in the bovine and ovine, because blastocysts have passed the critical step of genomic activation and have high developmental potential [19]. An efficient method of blastocyst cryopreservation for laboratory animals could be potential model for farm animals. Vitrification solutions containing different cryoprotectants have been used to cryopreserve animal blastocysts. Some researchers have reported that a mixture containing EG and DMSO can be used to successfully vitrify mouse blastocysts [20]. Supplementation with macromolecular reagent can reduce the toxicity of the solution by decreasing the concentration of the permeating agent required for vitrification. When Ficoll and sucrose (nonpermeating cryoprotectants) were incorporated into an EG-containing VS, Zhu et al. [15] obtained high viability of vitrified-warmed mouse blastocysts. With the invention of the OPS method, some researchers have successfully cryopreserved blastocysts from various mammalian species using a VS consisting of EG, DMSO, and sucrose (EDS) $[21,22]$. The present study investigated vitrification solutions containing the permeating cryoprotectants ED or EG + DMSO, small saccharide, and the macromolecular agent Ficoll 70. Mouse blastocysts were successfully vitrified with the EDFS30 or EFS40 solutions and thawed by the conventional procedure; in vitro development of the embryos showed no significant difference from those of the controls. Therefore, EDFS30 was selected for further experiments because it produced the highest in vitro survival of vitrifiedwarmed embryos within this group.
In-straw dilution and direct transfer has been used for bovine embryos frozen using the conventional procedure [23], which uses the concentration of cryoprotectants at a relatively low concentration. However, if embryos are cryopreserved by vitrification, the volume of VS in the straw must be as small as possible to avoid toxic effects on the further development of the embryos. In the experiments of Vajta et al. [6] and EldridgePanuska et al. [11], relatively large amounts of holding medium and small amounts of VS solution were loaded in a $0.25 \mathrm{ml}$ straw to decrease the VS concentration after dilution. Although previous reports have focused on in-straw dilution and direct transfer of embryos vitrified by the OPS method [14, 15], some factors, such as the volume of VS in the OPS and the immersion time in PBS, which may affect the viability of thawed embryos, have not been studied. Our results showed that the volume of VS in the OPS has no influence on the viability of post-thawed embryos when the length designating to the volume of VS in the OPS is shorter than $1 \mathrm{~cm}$. However, when the length designating to the volume of VS in the OPS is longer than $1.2 \mathrm{~cm}$, the viability of post-thawed embryos is significantly decreased. In this case, the VS cannot be diluted sufficiently because not enough MS enters the OPS by capillary action, and this results in lower development of in-straw thawed embryos.

In-straw dilution of embryos vitrified by the OPS method does not resemble conventional thawing in terms of the process of removing cryoprotectants. When the OPS-vitrified embryos were thawed by stepwise in-straw dilution, the VS in the OPS was diluted gradually with two different concentrations of sucrose solution and by PBS through capillary action. Sucrose has been found to be potentially detrimental to the viability of embryos at high concentrations and high temperatures [24, 25], although sucrose is virtually non-toxic when used 
at refrigerated temperatures [26]. Meanwhile, permeating agents have a strong toxic effect on embryos, and this effect would increase the longer embryos are exposed to these chemicals [27]. In the present experiment, embryos survived and maintained high developmental potential for up to 12 min in OPSs after thawing. This high developmental potential could mainly result from stepwise in-straw dilution with different concentrations of sucrose solution. During the dilution procedure, permeating agents may be removed gradually from the vitrified embryos, and osmotic swelling may be avoided to some extent.

The vitrified embryos survived in OPSs for up to $17 \mathrm{~min}$ after removal from liquid nitrogen (Table 3). This amount of time (17 $\mathrm{min})$ is sufficient for nonsurgical embryo transfer in the bovine, as this normally takes 3 to $8 \mathrm{~min}$ to accomplish based on our experience. So, in-straw thawing is fit for embryo transfer in the field. Our results indicate that the pregnancy and birth rates of the in-straw dilution and direct transfer groups were no different from those of the fresh control and conventional thawing groups. The birth rate $(40 \%)$ was comparable to previous reports $[19,28]$. In our experiment, all embryos were recovered after warming and dilution by immersing the OPSs in PBS for different amounts of time. One possible explanation for this phenomenon was that once an
OPS was plunged in diluent, the column of vitrified medium began to thaw immediately and the embryos were moved upward by capillary action. So far, the OPS method has been successfully applied to vitrify embryos from the ovine [22, 29], hircine [30], and porcine [31]. In terms of in-straw dilution and direct transfer of vitrified embryos, the OPS method has more advantages, such as a simpler procedure and higher post-thawing viability compared with the straw method [9]. Our results demonstrate that mouse embryos vitrified by the OPS method could be successfully thawed by the stepwise in-straw method under microscope free conditions and transferred directly to recipients. In summary, we established a simple and effective method of thawing mouse embryos vitrified by the OPS method, and this method may be a model for manipulation of vitrified embryos from small ruminants and swine under field conditions.

\section{Acknowledgements}

The present work was sponsored by the National "863" Program (project grant: 2004AA213071) and Beijing Agricultural Technology Program (project grant: H022020060420).

\section{References}

1. Kasai M. Vitrification: refined strategy for the cryopreservation of mammalian embryos. J Mamm Ova Res 1997; 14: 17-28.

2. Vajta G. Vitrification of the oocytes and embryos of domestic animals. Anim Reprod Sci 2000; 60-61: 357364.

3. Kuleshova LL, Lopata A. Vitrification can be more favorable than slow cooling. Fertil Steril 2002; 78: 449-454.

4. Kharche SD, Sharma GT, Majumdar AC. In vitro maturation and fertilization of goat oocytes vitrified at the germinal vesicle stage. Small Rumin Res 2005; 57: 81-84.

5. Berthelot F, Martinat-Botte F, Locatelli A, Perreau C, Terqui M. Piglets born after vitrification of embryos using the open pulled straw method. Cryobiology 2000; 41: 116-124.

6. Vajta G, Booth PJ, Holm P, Greve T, Callesen H. Successful vitrification of early stage bovine in vitro produced embryos with the open pulled straw
(OPS) method. Cyryo-letters 1997; 18: 191-195.

7. Pugh PA, Tervit A, Niemann H. Effects of vitrification medium composition on the survival of bovine in vitro produced embryos, following in straw-dilution, in vitro and in vivo following transfer. Anim Reprod Sci 2000; 58: 9-22.

8. Vicente JS, García-Ximénez F. Direct transfer of vitrified rabbit embryos. Theriogenology 1996; 45 : 811-815.

9. Baril G, Traldi AL, Cognié Y, Leboeu B, Beckers JF, Mermillod P. Successful direct transfer of vitrified sheep embryos. Theriogenology 2001; 56: 299-305.

10. Okada A, Yoshii K, Mizuochi Y, Andoh T, Joujou S, Wachi S, Togawa M, Ishida N, Asada M, Fukui Y. Viability of cyropreserved and vitrified embryos and fertility after direct transfer in ewes. $J$ Reprod Dev 2002; 48: 189-195.

11. Eldridge-Panuska WD, di Brienza VC, Seidel GE Jr, Squires EL, Carnevale EM. Establishment of pregnancies after serial dilution or direct transfer by 
vitrified equine embryos. Theriogenology 2005; 63: 1308-1319.

12. Isachenko V, Alabart JL, Dattena M, Nawroth F, Cappai P, Isachenko E, Cocero MJ, Olivera J, Roche A, Accardo C, Krivokharchenko A, Folch J. New technology for vitrification and field (microscope-free) warming and transfer of small ruminant embryos. Theriogenology 2003; 59: 1209 1218.

13. Cuello C, Berthelot F, Martinat-Botté F, Venturi E, Guillouet P, Vázquez JM, Rocaa Martínez EA. Piglets born after non-surgical deep intrauterine transfer of vitrified blastocysts in gilts. Anim Reprod Sci 2005; 85: 275-286.

14. Martino A, Songsasen N, Leibo SP. Development into blastocysts of bovine oocytes cryopreserved by ultra-rapid cooling. Biol Reprod 1996; 54: 1059-1069.

15. Zhu SE, Kasai M, Otoge H, Sakurai T, Machida T. Cryopreservation of expanded mouse blastocysts by vitrification in ethylene glycol based solutions. I Reprod Fertil 1993; 98: 139-145.

16. Kasai M, Komi JH, Takakamo A, Tsudera H, Sakurai T, Machida T. A simple method for mouse embryo cryopreservation in a low toxicity vitrification solution without appreciable loss of viability. J Reprod Fertil 1990; 89: 91-97.

17. Zhou GB, Zhu SE, Hou YP, Jin F, Yang QE, Yang ZQ, Quan GB, Tan HM. Vitrification of mouse embryos at various stages by open-pulled straw (OPS) method. Anim Biotechnol 2005; 16: 153-163.

18. Chatot CL, Ziomek CA, Bavister BD, Leais JL, Torres I. An improved culture medium supports development of random-bred 1-cell mouse embryos in vitro. J Reprod Fertil 1989; 86: 679-688.

19. Ménézo YJ. Blastocyst freezing. Euro J Obstet Gynecol Reprod Biol 2004; 115 (Suppl 1): S12-15.

20. Ishimori $\mathbf{H}$, Takahashi $\mathbf{Y}$, Kanagawa $\mathbf{H}$. Viability of vitrified mouse embryos using various cryoprotect mixtures. Theriogenology 1992; 37: 481-487.

21. Kong IK, Lee SI, Cho SG, Cho SK, Park CS. Comparison of open pulled straw(ops) vs glass micropipette (GMP) vitrification in mouse blastocysts. Theriogenology 2000; 53: 1817-1826.

22. Dattena M, Accardo C, Pilichi S, Isachenko V, Mara L, Chessa B, Cappai P. Comparison of different vitrification protocols on viability after transfer of ovine blastocysts in vitro produced and in vivo derived. Theriogenology 2004; 62: 481-493.

23. Hasler JF. The current status and future of commercial embryo transfer in cattle. Anim Reprod Sci 2003; 79: 245-264.

24. Kuleshova LL, MacFarlane DR, Trounson AO, Shaw JM. Sugars exert a major influence on the vitrification properties of ethylene glycol-based solutions and have low toxicity to embryos and oocytes. Cryobiology 1999; 38: 119-130.

25. Pedro PB, Sakurai T, Edashige K, Kasai M. Effects of osmotic shrinkage on the survival of mouse oocytes and embryos at various developmental stages. J Mamm Ova Res 1997; 14: 66-71.

26. Kasai M. Nonfreezing technique for short-term storge of mouse embryos. I In Vitro Fert Embryo Transf 1986; 3: 10-14.

27. Kasai M, Ito K, Edashige $\mathbf{K}$. Morphological appearance of the cryopreserved mouse blastocysts as a tool to identify the type of cryoinjury. Hum Reprod 2002; 17: 1863-1874.

28. Nowshari MA, Brem G. Effect of cryoprotectants and their concentration on post-thaw survival and development of expanded mouse blastocysts frozen by a simple rapid-freezing procedure. Theriogenology 1998; 50: 1001-1013.

29. Papadopoulos S, Rizos D, Duffy P, Wade M, Quinn K, Boland MP, Lonergan P. Embryo survival and recipient pregnancy rates after transfer of fresh or vitrified, in vivo or in vitro produced ovine blastocysts. Anim Reprod Sci 2002; 74: 35-44.

30. EI-Gayar M, Holtz W. Technical note: vitrification of goat embryos by the open pulled-straw method. J Anim Sci 2001; 79: 2436-2438.

31. Berthelot F, Martinat-Botte F, Vajta G, Terqui M. Cryopreservation of procine embryos: state of the art. Livest Prod Sci 2003; 83: 73-83. 